\title{
Should prospective dental students be screened for colour vision deficits?
}

\author{
F. Mushtaq, ${ }^{1}$ R. C. Baraas, ${ }^{2}$ L. M. Al-Saud,${ }^{3}$ I. Mirghani, ${ }^{3}$ C. van der Zee, ${ }^{3}$ E. Yates, ${ }^{5}$ A. Keeling, ${ }^{3}$ \\ M. A. Mon-Williams ${ }^{* 1,2,4}$ and M. Manogue ${ }^{3}$
}

In Brief

Describes the issues inherent in assessing prospective dental students
Outlines one specific problem encountered in recent assessmentcolour vision deficiency (CVD).
Provides a comprehensive overview of the role of CVD in dentistry.
Discusses compensatory strategies for practising clinicians.

A perennial question in dental education is: what skills and aptitudes should be assessed in prospective dental students? Intellectual capacity and manual dexterity understandably rank highly, but are there minimum thresholds for visual perception that applicants need be able to demonstrate before they enter the profession? We have recently flagged this issue with regard to the thresholds of stereoscopic acuity required for a dentist when operating on teeth. In the present article, we highlight the issue of identifying a minimum acceptable level of colour vision.

Dental schools are increasingly adopting 'Multiple Mini Interview' (MMI) approaches to evaluate prospective students. But what skills and abilities should be assessed within these short, structured 'interview' stations? There has been a general agreement that skills related to problem solving and communication skills should be included. There has also been a desire to assess the perceptual-motor abilities of the dental applicants - because numerous dental tasks require 'good hand-eye coordination'. Nevertheless, there is no clear understanding of what constitutes an acceptable level of motor skill in dentistry. It is obviously the case that gross physical disability would preclude entrance to the profession (an inability to physically hold dental instruments would be an insurmountable barrier), but it is harder to determine at what point subtle

${ }^{1}$ School of Psychology, Faculty of Medicine and Health, University of Leeds, Leeds, West Yorkshire; ${ }^{2}$ Department of Optometry and Visual Science, National Centre for Optics, Vision and Eye Care, University College of Southeast Norway, Kongsberg, Norway; ${ }^{3}$ School of Dentistry, Faculty of Medicine and Health, University of Leeds, Leeds, West Yorkshire; ${ }^{4}$ Bradford Institute of Health Research, Bradford Teaching Hospitals NHS Trust, Bradford; ${ }^{5}$ School of Dentistry, The University of Western Australia, Crawley, Australia ${ }^{*}$ Correspondence to: Mark Mon-Williams Email: m.mon-williams@leeds.ac.uk

Refereed Paper. Accepted 4 July 2016

DOI: 10.1038/sj.bdj.2016.633

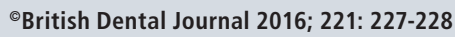

motor deficits become a barrier to achieving an acceptable skill level. These issues require empirical examination and this is a major component of our current research, and one reason why we assess the motor skills of all dental applicants to the University of Leeds.

Our assessment of motor skill in dental applicants recently raised another issue regarding the entrance requirements of prospective students - viz., the minimum thresholds for visual perception. In the same way that gross physical disability prevents access to the dental profession, the gross perceptual disability caused by blindness precludes an individual from practising dentistry (for obvious reasons). Once more an extreme example highlights the difficulty in identifying minimum acceptable functional levels of human ability (in this case, level of vision). We recently flagged this issue with regard to the thresholds of stereoscopic acuity required for a dentist when operating on teeth. ${ }^{1}$ In the present article, we highlight the issue of identifying a minimum acceptable level of colour vision.

We became aware of the issue of colour vision deficit in dental applicants following a recent series of MMIs. One of the stations adapted a validated objective measure of manual control to assess the applicants' motor abilities. ${ }^{2,3}$ The test was delivered on a touch screen tablet using a stylus pen and applicants were asked to use the stylus to move from a starting location (coloured white, red or green) to one of three different coloured end points (white, red and green) at the other end of the screen. The aim was to move from the starting location to the endpoint target as quickly and accurately as possible. The colour of the starting location indicated which target was the endpoint location. From the 348 candidates, five found the task to be extremely challenging as a result of their self-reported colour vision deficiency (CVD). This raised the question of whether these applicants should be precluded from entering the dental profession and whether colour vision should be tested as a matter of course as part of the entrance requirements - an issue that we believe requires urgent debate.

In a typically functional visual system, there are three different classes of cone photoreceptors (blue, green and red, also known as short, medium and long wavelength sensitive, respectively). Each contains a different type of photopigment, which are maximally responsive to light of varying wavelengths. To derive normal colour vision, the human visual system compares and combines the response of the different cones to light. Colour perception, including discrimination and matching, also depends on the physical properties of the light shining onto the fixated objects, the surface properties of the object under scrutiny and surrounding objects. The latter cannot be 
ignored as perceived colour (its appearance) is influenced by the chromaticity of its surround. ${ }^{4}$ CVD, in its congenital form, is the product of genetic mutations that affect the expression of the three cone types and is an extremely common vision disorder - mainly affecting males (on the X chromosome within the Xq28 band) with incidence rates of approximately $8 \%$ for males and $0.5 \%$ for females. Unfortunately, CVD cannot be improved with training and, to date, there is no treatment (although gene therapy shows promise). ${ }^{5}$

There is evidence to suggest that colour vision is integral to a number of dental specialties (particularly restorative and prosthetic) and specific tasks (colour shade matching). ${ }^{6,7}$ As aesthetic dentistry becomes increasingly popular, dentists are required to ensure that modifications to teeth provide as close a match as possible to a patient's own teeth in terms of size, shape and colour. ${ }^{8}$ However, it has been shown that dentists with CVD are more likely to have errors in hue and chroma (although interestingly not in value) selection ${ }^{9}$ and red-green deficient dental professionals can be severely affected by lighting conditions and colour temperature. ${ }^{6}$

Team-based approaches to shade matching could help a CVD dentist in achieving a more precise shade selection. ${ }^{9,10}$ An additional compensatory strategy is an increased use of photographs to communicate shade (in addition to characterisation) to the laboratory. While this might mitigate the impact of the issue of CVD in the clinic, the emphasis then shifts to technicians. It is worth noting that current (for example, digital shade guides) and upcoming (for example, smartphone devices) technologies have the potential to negate the effect of CVD in the clinic, whilst much progress has been made in manufacturing colour consistent 'blanks' for CADCAM dentistry, the final characterisation of all highly aesthetic restorations must currently be finished by hand (and will be thus be dependent on the artistic skills of the technician). It may be that colour vision screening becomes increasingly more pertinent to the technician's role than the dentist.

The issue of precluding an applicant on the basis of a physical impairment is inherently thorny. On the one hand, there are strong moral arguments to be made regarding the need for inclusivity within society regardless of whether an individual has physical or mental impairment. On the other hand, it is clearly undesirable to allow practitioners to provide a professional service when they lack the requisite core skills. We note that there is little controversy about preventing individuals with poor examination results (which at least sometimes must indicate a lack of cognitive capability) from entering the dental profession. It may be that it is easier to see the links between poor academic performance and an inability to cope with the demands of dental education. In contrast, it is harder to understand how a colour vision deficit will impact on an individual's ability to work as a dentist. Thus, the question of whether a colour vision deficit should preclude admission is highly vexed. There are arguments to support this approach (under the premise that decreasing patient risk is paramount within any profession) until it has been established that such deficits are not problematic (or can be managed successfully). The counter argument is that it should be first established that the deficit does cause a problem before such a criterion is added to the admittance requirements. We argue that urgent work is required in either situation in order to empirically determine the relationship between colour vision deficit and dental competence.

It is worth highlighting the ease of screening for colour vision deficiencies using pseudoisochromatic plate tests. When tested under standardised illumination, these relatively cheap, fast, and simple to use methods produce good results. The most commonly used sets are the Ishihara plates (detecting red-green deficits) and the Richmond HRR (Hardy, Rand and Rittler) plates ${ }^{11}$ - which include cards for blue-yellow (tritan) deficiencies and are also suitable for acquired colour vision defects. However, it must be noted that the ability to grade the severity of colour-vision defects with pseudoisochromatic test plates is limited. ${ }^{12,13}$ and results from these tests cannot yet be used as a basis for selecting individuals who should be allowed to become dentists. To illustrate, there is large between-individual variability in the perception of white and a very large range of colours are perceived as white by the same observers on different occasions. The variation of white is more affected by the processing of colours along the blue-yellow direction, rather than the red-green direction. ${ }^{14-16}$ Thus, screening for red-green vision deficiencies with the pseudoisochromatic test alone will only serve to inform prospective students if it is likely that they have CVD. Some red-green colour deficient individuals, however, perform as well as those with normal colour vision when discriminating colours under test conditions that are more naturalistic than standardised tests for colour vision deficiencies. ${ }^{16,19}$
We conclude by noting that the railway industry faced such debates about colour vision deficits in the previous century. In the end, a practical and humane solution was found where initial screening for CVD was followed up by functional task-related tests of vision. The solution was to check for CVDs, but only preclude an individual from driving trains if the CVD was found to limit the ability to detect stop signals. The emergence of virtual reality simulators might provide the dental profession with good functional tasks that will allow an assessment of the functional impact of a CVD detected through initial screening. ${ }^{17,18}$ At the very least, identifying and informing students with CVD should allow for the candidate to receive the necessary support and training to pursue a successful dental career.

1. Mon-Williams M, Mushtaq F, Wilkie R M, Khambay B, Manogue M. A three dimensional view of stereopsis in dentistry. Br Dent J 2015; 219: 26-27.

2. Flatters I, Mushtaq F, Hill L J B et al. Children's head movements and postural stability as a function of task. Exp Brain Res 2014; 232: 1953-1970.

3. Culmer PR, Levesley M C, Mon-Williams M, Williams JHG. A new tool for assessing human movement: the kinematic assessment tool. J Neurosci Methods 2009; 184: 184-192.

4. Shevell S K, Kingdom F A A. Colour in complex scenes key words. Annu Rev Psychol 2008; 59: 143-166.

5. Neitz M, Neitz J. Curing colour blindness-mice and non human primates. Cold Spring Harb Perspect Med 2014; 4 a017418-a017418.

6. Gokce H S, Piskin B, Ceyhan D, Gokce S M, Arisan V. Shade matching performance of normal and colour vision-deficient dental professionals with standard daylight and tungsten illuminants. J Prosthet Dent 2010; 103: 139-147.

7. Ethell J, Jarad F D, Youngson C C. The effect of colour defective vision on shade matching accuracy. Eur J Prosthodont Restor Dent 2006; 14: 131-136.

8. Naik A V, Pai R C. Colour blindness in dental students and staff an obstacle in shade selection for restorations. Ann Essences Dent 2010: 2: 25-28.

9. Davison S P, Myslinski N R. Shade selection by colour vision-defective dental personnel. J Prosthet Dent 1990; 63: 97-101.

10. Wasson W, Schuman N. Colour vision and dentistry. Quintessence Int 1992; 23: 349-353.

11. Cole B L, Lian KY, Lakkis C. The new Richmond HRR pseudoisochromatic test for colour vision is better than the Ishihara test. Clin Exp Optom 2006; 89: 73-80.

12. Barbur J L, Rodriguez-Carmona M. 2 - Variability in normal and defective colour vision: consequences for occupational environments. In J Best (Ed). Colour Design. pp 24-82. Elsevier. 2012.

13. Cole B L. Assessment of inherited colour vision defects in clinical practice. Clin Exp Optom 2007; 90: 157-175.

14. Webster M A, Leonard D. Adaptation and perceptual norms in colour vision. J Opt Soc Am A Opt Image Sci Vis 2008: 25: 2817-2825.

15. Werner J S, Walraven J. Effect of chromatic adaptation on the achromatic locus: The role of contrast, luminance and background colour. Vision Res 1982; 22: 929-943.

16. Baraas R C, Foster D H, Amano K, Nascimento S M C. Anomalous trichromats' judgements of surface colour in natural scenes under different daylights. Vis Neurosci 2006; 23: 629-635.

17. Al-Saud L M, Mushtaq F, Allsop M J et al. Feedback and motor skill acquisition using a haptic dental simulator. Eur J Dent Educ 2016; 1-8.

18. Mirghani I, Mushtaq F, Allsop M, Potter C, Tickhill N, Al-Saud LM $S$ et al. Capturing differences in dental training using a virtual reality simulator. (Accepted pending minor revisions). Eur J Dent Educ 2016. 